\title{
Current instability and diamagnetism in small-diameter carbon nanotubes
}

\author{
J. González \\ Instituto de Estructura de la Materia, Consejo Superior de Investigaciones Científicas, Serrano 123, 28006 Madrid, Spain
}

(Received 4 March 2005; revised manuscript received 27 June 2005; published 11 August 2005)

\begin{abstract}
We investigate the electronic instabilities in carbon nanotubes of short radius, looking at the scaling behavior of the different correlations and charge stiffnesses at low energies. We show that the Luttinger liquid behavior breaks down in the undoped $(3,3)$ nanotubes at low temperatures, due to the appearance of soft modes in the sector of current excitations. The phenomenology derived from this regime is consistent with the development of a gap in the single-particle spectrum as well as with a divergent diamagnetic susceptibility, as observed in the experiments carried out in carbon nanotubes of small diameter.
\end{abstract}

DOI: 10.1103/PhysRevB.72.073403

PACS number(s): 71.10.Pm, 71.20.Tx, 74.50.+r

Carbon nanotubes $(\mathrm{CN})$ are among the best candidates for the development of devices in molecular electronics. For this reason, there has been great interest during the past years in the investigation of their electronic properties. As the electrons are constrained to move in the reduced dimensions of the tubule, $\mathrm{CN}$ constitute a paradigm of strongly correlated electron system. ${ }^{1-4}$ Thus, it has been possible to observe signatures of Coulomb blockade in short nanotube samples, as well as signatures of one-dimensional (1D) transport characteristic of Luttinger liquid behavior. ${ }^{5,6}$

Yet there has been also clear evidence of superconducting (SC) correlations in $\mathrm{CN}$ attached to suitable contacts. ${ }^{7,8} \mathrm{Su}-$ percurrents have been observed in the samples with SC electrodes reported in Ref. 7, providing evidence of the proximity effect in the CN. ${ }^{9}$ Moreover, SC transitions have been measured in nanotube ropes attached to highly transparent contacts. ${ }^{10}$ More recently, measurements carried out in $\mathrm{CN}$ inserted in a zeolite matrix have supported the conclusion that strong SC correlations should exist in $\mathrm{CN}$ of very short diameter, leading to a transition temperature $T_{c} \sim 15 \mathrm{~K} .{ }^{11}$

The experiment reported in Ref. 11 has provided evidence of the diamagnetic behavior of the $\mathrm{CN}$ at low temperatures. From inspection of the $I-V$ characteristics, it has also appreciated the appearance of a gap in the single-particle spectrum, which has been used to infer the value of $T_{c}{ }^{11}$ However, the lack of evidence for a true SC transition stresses the fact that the above-mentioned observations refer to properties of the individual $\mathrm{CN}$, which are not coupled by electron hopping as they only have a weak interaction with the zeolite walls. ${ }^{11}$ In general, the tunneling amplitude between metallic nanotubes is what dictates the setting of 3D Cooper-pair coherence and the value of $T_{c}{ }^{12,13}$ The existence of a transition scale intrinsic to the individual $\mathrm{CN}$ demands therefore a reexamination of the role of the dominant correlations in nanotubes of short radius.

The nanotube diameter $d=4.2 \pm 0.2 \AA$ reported in Ref. 11 is closer to the value calculated for a $(3,3)$ nanotube geometry, although the presence of $(5,0)$ nanotubes in the zeolite matrix cannot be discarded. ${ }^{14}$ It has been shown by means of the local-density functional method that the $(3,3)$ nanotubes have the same band structure of typical armchair nanotubes near the Fermi level, with a pair of subbands crossing at two opposite momenta. ${ }^{14}$ The case of the $(5,0)$ nanotubes is different in that they have one more subband crossing the Fermi level, with angular momentum $L=0$ in the zigzag geometry. We will apply in what follows a scaling analysis to discern the low-energy electronic instabilities in these two types of nanotubes.

Focusing first on the $(3,3)$ nanotubes, the interaction processes between different low-energy branches crossing at Fermi points $k_{F}$ and $-k_{F}$ can be classified by attaching respective coupling constants $g_{i}^{(j)}$ to them. ${ }^{15}$ The lower index discerns whether the interacting particles shift from one Fermi point to the other $(i=1)$, remain at different Fermi points $(i=2)$, or if they interact near the same Fermi point $(i=4)$. The upper label follows the same rule to classify the different combinations of left and right movers, including the possibility of having umklapp processes $(j=3)$ in the undoped system.

While the effect of the different interactions will be considered through the analysis of their low-energy scaling, it becomes convenient to adopt a bosonization approach to deal with the interactions in forward-scattering (FS) channels. Thus, we first introduce an electron density operator $\rho_{r i}(k)$ for each of the linear branches of the subbands crossing the Fermi level, where the index $r=L, R$ denotes the left- or right-moving character and the index $i=1,2$ labels the Fermi point. It becomes possible to decouple the FS channels by passing to the combinations

$$
\rho_{r \pm}(k)=\nu\left[\rho_{r 1}(k) \pm \rho_{r 2}(k)\right] / \sqrt{2},
$$

where $\nu=-1$ for $\rho_{L_{-}}$and +1 otherwise. The Hamiltonian for the FS interactions can be written in terms of fields $\Phi_{ \pm}(x)$, such that $\partial_{x} \Phi_{ \pm}(x)=\sqrt{\pi}\left[\rho_{L \pm}(x)+\rho_{R \pm}(x)\right]$, and their conjugate momenta $\Pi_{ \pm}(x)$,

$$
H_{F S}=\frac{1}{2} \int d x \sum_{s= \pm}\left\{v_{J s}\left[\Pi_{s}(x)\right]^{2}+v_{N s}\left[\partial_{x} \Phi_{s}(x)\right]^{2}\right\} .
$$

The four independent velocities are given by

$$
\begin{aligned}
& v_{J \pm}=v_{F}+(1 / \pi)\left[g_{4}^{(4)} \pm g_{2}^{(4)}-\left(g_{2}^{(2)} \pm g_{4}^{(2)}\right)\right], \\
& v_{N \pm}=v_{F}+(1 / \pi)\left[g_{4}^{(4)} \pm g_{2}^{(4)}+\left(g_{2}^{(2)} \pm g_{4}^{(2)}\right)\right],
\end{aligned}
$$

where $v_{F}$ is the Fermi velocity. In terms of these quantities, the renormalized velocities of the liquid are $u_{ \pm}=\sqrt{v_{N_{ \pm}} v_{J_{ \pm}}}$ 
while the charge stiffnesses are $K_{ \pm}=\sqrt{v_{J_{ \pm}} / v_{N_{ \pm}}} \cdot 16$

In general, the significance of the interactions is given by their scaling behavior at low energies. The scaling equations of the $g_{i}^{(j)}$ couplings have been obtained in Ref. 15 for an armchair geometry. Here we have introduced a nonperturbative improvement of the equations, writing the scaling dimensions $\Delta_{i j}$ of the couplings in terms of the $K_{ \pm}$parameters, and we have introduced the pertinent modifications for the $(5,0)$ geometry. The equations take in general the form

$$
\frac{\partial g_{i}^{(j)}}{\partial l}=\Delta_{i j}\left(K_{ \pm}\right) g_{i}^{(j)}+\frac{1}{\pi v_{F}} \sum\left(c_{p q, m n} g_{p}^{(q)} g_{m}^{(n)}+c_{k l} u_{k} u_{l}\right)
$$

where $l$ stands for minus the logarithm of the energy (temperature) scale measured in units of the high-energy scale $E_{c}$ of the 1D model (of the order of $\sim 0.1 \mathrm{eV}$ ). The $g_{i}^{(3)}$ couplings only arise in the $(3,3)$ nanotubes without doping (which is the likely experimental condition when embedded in the insulating zeolite matrix) while the additional $u_{F}$ and $u_{B}$ couplings (to be defined later) apply only to the $(5,0)$ nanotubes.

The scaling equations have to be solved with initial conditions accounting for the competition between the Coulomb interaction and the effective interaction arising from phonon exchange. ${ }^{17}$ The latter turns out to be attractive in the backscattering (BS) channels and repulsive in the umklapp channels. ${ }^{12,18}$ Its effective coupling $g$ has a strength $\approx 0.3-0.9$ times $v_{F} / n$ for a $(n, n)$ nanotube. ${ }^{18}$ This is comparable to the strength of the Coulomb interaction in BS and umklapp processes, which we have taken as $\approx 0.2 e^{2} / n$ following Refs. 2 and 3. Given that $e^{2} \approx 2.7 v_{F}$, the Coulomb repulsion only becomes dominant in forward-scattering processes mediated by the potential $V(q) \approx\left(2 e^{2} / \kappa\right) \log (1$ $+q_{0} / q$ ), where $q$ is the momentum and $\kappa$ a suitable dielectric constant. ${ }^{19}$ Under these initial conditions, the scaling equations develop an unstable flow below certain energy scale, at which the $g_{2}^{(1)}$ and $g_{4}^{(1)}$ couplings enter a regime of large attraction while the $g_{4}^{(2)}$ and umklapp couplings are driven towards large repulsion in the $(3,3)$ nanotubes.

We have discerned the character of the electronic instability by looking for the susceptibility with the fastest growth as $l \rightarrow \infty$, as well as for the possible breakdown of the Luttinger liquid parameters $K_{ \pm}$. We have checked that this latter instance takes place before any charge-density-wave (CDW) or pairing response function starts to diverge in the $(3,3)$ nanotubes. Their phase diagram is represented in Fig. 1. The strength of the Coulomb potential $V$ for the samples reported in Ref. 11 has to be determined by taking into account the screening effects from the large 3D array of $\mathrm{CN}$ inserted in the zeolite channels. These effects can be studied by generalizing the many-body approach devised in Ref. 20. Thus, by performing a RPA analysis incorporating the electrostatic coupling between all the $\mathrm{CN}$ in the array, we have found that the Coulomb potential ranges from $V \approx 0.5 e^{2}$ at $q$ $=10 \mathrm{~nm}^{-1}$ to a saturation value $V \approx 0.9 e^{2}$ for $q=0.1 \mathrm{~nm}^{-1}$. Taking $e^{2} \approx 2.7 v_{F}$ and $|g| \approx 0.2-0.3 v_{F}$ for the $(3,3)$ nanotubes, we observe that the relevant regime corresponds to the phase $K_{+}=0$, with very weak SC or CDW correlations.
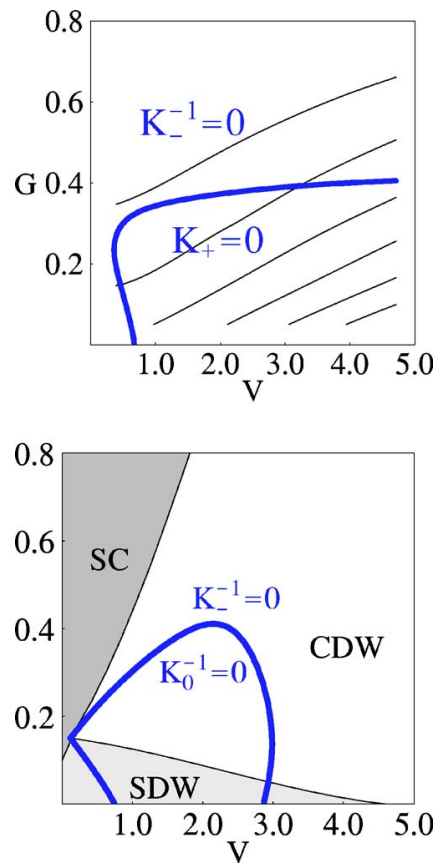

FIG. 1. (Color online) Phase diagram of $(3,3)$ (top) and $(5,0)$ (bottom) $\mathrm{CN}$ in terms of the Coulomb potential $V$ (in units of $v_{F}$ ) and the effective coupling of the phonon-exchange interaction $G$ $=4|g| / \pi v_{F}$. The thick lines are boundaries between the different phases characterized by the breaking of the parameters $K_{ \pm}$and $K_{0}$ at low temperature scales. In the $(3,3)$ nanotubes, CDW correlations at zero momentum are dominant at the singular point, but with low strength represented by the contours of constant CDW response function $R=2,4,8,16,32,64$ (from top to bottom). For the $(5,0)$ nanotubes, three different regimes arise with strong correlations at the singular point of $K_{-}$or $K_{0}$, given by the dominance of the $2 k_{F}$ CDW (white area), the $2 k_{F}$ spin-density wave (light area), or the $s$-wave SC response function (dark area).

A phase with $K_{+}=0$ has been already described in the study of $\mathrm{CN}$ with purely repulsive interactions. ${ }^{4}$ This instance has been also analyzed in Ref. 21. In the case of $\mathrm{CN}$ of short radius, however, we have a non-negligible strength of the phonon-exchange interaction and larger contributions to BS and umklapp interaction channels. This leads to the appearance of an electronic instability at a much larger temperature than for nanotubes of typical radius. The physical situation is also different in that the vanishing of $v_{J_{+}}$in (3) is what triggers the instability, while the compressibility $K_{+} / u_{+}$ remains nonvanishing at the point of the transition.

The onset of the phase characterized by the vanishing of $K_{+}$may explain the apparent gap that has been measured from the $I-V$ curves in Ref. 11. In our description, the depletion of the density of states $n(\varepsilon)$ near the Fermi level can be evaluated by means of bosonization methods, with the result that

$$
n(\varepsilon) \sim \varepsilon^{\left(K_{+}+1 / K_{+}+K_{-}+1 / K_{-}-4\right) / 8},
$$

where $\varepsilon$ is measured in units of the cutoff $E_{c}$. The approach to the ground-state properties of the system is achieved by following the scaling of the $K_{+}, K_{-}$parameters for increasing values of $l$. In this respect, we take the logarithm of the 


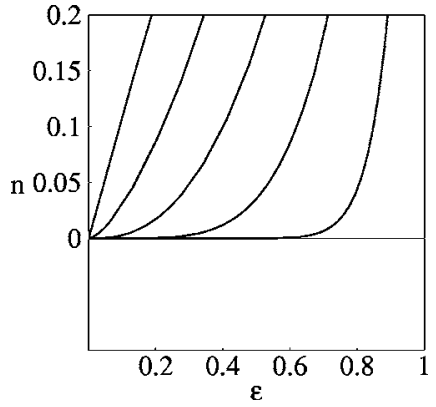

FIG. 2. Plot of the density of states $n$ as a function of the energy $\varepsilon$ (in units of $E_{c}$ ) for $V=0.75 \pi v_{F}$ and $4|g| / \pi v_{F}=0.2$. The curves correspond to values of minus the logarithm of the temperature scale $l=3.21,3.225,3.232,3.235$, and 3.236.

inverse of the thermal energy $k_{B} T$ (in units of $E_{c}$ ) as a measure of the variable $l$ in the flow of the scaling equations. We observe that the density of states follows a power-law behavior with an increasingly large exponent as $K_{+}$vanishes in the low-temperature limit, as represented in Fig. 2. For the above estimates of the interaction strengths, the temperature $T_{c}$ at which $K_{+}$vanishes is given by values of $\ln \left(E_{c} / k_{B} T_{c}\right)$ in the range of 3-4. This corresponds to values of $T_{c}$ of the order of $\sim 10 \mathrm{~K}$. There is a more accelerated development of the gap in comparison to the experimental data, which may be a consequence of relying on the second-order perturbative scaling. We observe anyhow that the shapes of the curves obtained from (6) are in very good agreement with the form of the differential conductivity $d I / d V$ from the $I-V$ characteristics reported in Ref. 11.

Shifting now to the case of the $(5,0)$ nanotubes, their behavior can be studied in parallel to the above analysis. One has to incorporate an additional type of density operators for the subband with angular momentum $L=0$ (and Fermi velocity $v_{F}^{\prime}$ ), which has its own renormalized velocity $u_{0}$ and charge stiffness $K_{0}$. Moreover, there are new interaction processes in which one or two of the incoming modes belong to the subband with $L=0$. These interactions may be classified with an additional set of couplings $f_{i}^{(j)}$, where the indices $i$ and $j$ keep the same meaning as for the $g_{i}^{(j)}$ couplings. Finally, there may be processes in which two particles with opposite $L$ around the Fermi points 1 and 2 end up in the subband with $L=0$, requiring the introduction of new couplings $u_{F}$ and $u_{B}$ for the respective cases with and without change of chirality of the particles.

The processes given by the couplings $u_{F}$ and $u_{B}$ have been overlooked in other analyses of the $(5,0)$ nanotubes, ${ }^{22}$ while they are determinant in the enhancement of the SC correlations. Their scaling equations are given by

$$
\begin{aligned}
\frac{\partial u_{F}}{\partial l}= & \Delta u_{F}-\frac{1}{2 \pi v_{F}}\left[g_{1}^{(2)} u_{F}+\left(g_{2}^{(1)}+g_{1}^{(1)}+\beta f_{4}^{(1)}\right) u_{B}\right], \\
\frac{\partial u_{B}}{\partial l}= & \Delta u_{B}-\frac{1}{2 \pi v_{F}}\left[g_{1}^{(2)} u_{B}+\left(g_{2}^{(1)}+g_{1}^{(1)}+\beta f_{4}^{(1)}\right) u_{F}\right] \\
& +\frac{\alpha}{\pi v_{F}} f_{1}^{(1)}\left(u_{F}-2 u_{B}\right),
\end{aligned}
$$

where $\Delta=1-1 / 4 K_{+}-1 / 4 K_{-}-1 / 2 K_{0}+\alpha f_{2}^{(2)} / \pi v_{F}, \beta=v_{F} / v_{F}^{\prime}$, and $\alpha=2 /\left(1+v_{F}^{\prime} / v_{F}\right)$. The dominant contributions in these channels come from phonon-exchange processes, so that the interactions $u_{F}$ and $u_{B}$ enter a regime of large attraction at low energies. The flow of the scaling equations gives rise now to a fast growth of the $2 k_{F} \mathrm{CDW}$, the $2 k_{F}$ spin-density wave, or the $s$-wave SC response function, leading to the phases shown in Fig. 1. Recalling the theoretical estimates of the couplings for the experimental samples, we see that the relevant region falls in this case into the phase with strong CDW correlations.

An important point in connection with the phenomenology reported in Ref. 11 is that the $(3,3)$ nanotubes fall at low energies into a phase which is consistent with the large diamagnetic signal observed in the experiments. This can be seen by recalling that the vector part $\mathbf{A}$ of the electromagnetic potential couples to the electronic current. Actually, around each Fermi point, the modes of the electron field can be arranged into a bispinor $\Psi$, whose Hamiltonian is obtained from that of the low-energy excitations of graphene, ${ }^{23}$

$$
H_{G}=-i v_{F} \int d^{2} r \Psi^{\dagger}(\mathbf{r}) \sigma \cdot(\boldsymbol{\nabla}-i(e / c) \mathbf{A}) \Psi(\mathbf{r})
$$

For the purpose of evaluating the response to a transverse magnetic field, it is enough to consider the case of a slowly varying vector potential. The $1 \mathrm{D}$ projection to the lowenergy excitations of the nanotube can be done consistently by choosing the vector potential in the longitudinal direction of the nanotube. ${ }^{24}$ After diagonalizing the quadratic form in (9), we find that the vector potential introduces the additional contribution to the Hamiltonian

$$
H_{A}=v_{F}(e / c) \sum_{i} \int d x A_{\|}\left(\Psi_{L i}^{\dagger} \Psi_{L i}-\Psi_{R i}^{\dagger} \Psi_{R i}\right),
$$

where $\Psi_{L i}(x)$ and $\Psi_{R i}(x)$ are the electron fields for the respective linear branches around the two Fermi points $i$ $=1,2$. It becomes clear that the vector potential couples to the current $\Pi_{+}=\sqrt{\pi / 2}\left(\rho_{R 1}-\rho_{L 1}+\rho_{R 2}-\rho_{L 2}\right)$.

Considering the magnetic field as a small perturbation to the interacting electron system, the partition function $Z$ can be computed making use of the expansion

$$
Z=\left\langle e^{i S_{A}}\right\rangle=e^{\left[\left\langle i S_{A}\right\rangle-1 / 2\left(\left\langle S_{A}^{2}\right\rangle-\left\langle S_{A}\right\rangle^{2}\right)+\ldots\right]},
$$

where $S_{A}$ is defined by the time integral of $-H_{A}$, and the expectation values are taken in the interacting electron system. To second order in the vector potential, we obtain the contribution to the free energy $F$,

$$
F \approx v_{F}^{2}(e / c)^{2} \frac{1}{\pi} \int d k d \omega A_{\|}\left\langle\Pi_{+}(\omega, k) \Pi_{+}(-\omega,-k)\right\rangle A_{\|} .
$$

The response function for $\Pi_{+}$is ${ }^{16}$

$$
\left\langle\Pi_{+}(\omega, k) \Pi_{+}(-\omega,-k)\right\rangle=\frac{i}{K_{+}} \frac{u_{+} k^{2}}{\omega^{2}-u_{+}^{2} k^{2}} .
$$

Making the passage to imaginary frequency $\widetilde{\omega}=-i \omega$ and taking the static limit, we obtain the diamagnetic susceptibility 
$\sim v_{F}^{2}(e / c)^{2} / K_{+} u_{+}$, which displays a divergent behavior due to the vanishing of $K_{+}$and $u_{+}$at low temperatures, in agreement with the divergence of the magnetic susceptibility of $\mathrm{CN}$ reported in Ref. 11.

The preceding derivation shows that the large diamagnetic signal arises as a consequence of the enhanced susceptibility for the charge mismatch between left and right branches near the Fermi level. This effect can be clarified by focusing on the dependence of the Hamiltonian $H_{F S}$ for FS interactions on the fields $\Pi_{+}$and $\Phi_{+}$,

$$
H_{F S}=\frac{1}{2} \int d x\left(v_{J+} \Pi_{+}^{2}+v_{N+}\left(\partial_{x} \Phi_{+}\right)^{2}-\frac{2 \sqrt{2}}{\sqrt{\pi}} v_{F} \stackrel{e}{-} A_{\|} \Pi_{+}\right) .
$$

The vanishing of $v_{J_{+}}$at low temperatures in the $(3,3)$ nanotubes gives rise to the appearance of soft modes in the system. These correspond to the mismatch in the number of particles $N_{L}$ and $N_{R}$ at respective left and right branches in the dispersion relation. Disregarding fluctuations in the spectrum, we may approach $\Pi_{+} \approx \sqrt{\pi}\left(N_{R}-N_{L}\right){ }^{16}$ According to (14), very small energy is needed to transfer particles between left and right branches near the Fermi level as $v_{J_{+}}$ $\rightarrow 0$ and, under the influence of the magnetic field, we have

$$
N_{R}-N_{L} \approx \frac{\sqrt{2}}{\pi} \frac{v_{F}}{v_{J+}} \frac{e}{c} A_{\|}
$$

This enhanced susceptibility for current excitations is at the origin of the screening of the magnetic field, in close similarity to the conventional screening of the electric field by charge excitations.

To conclude, we end up with a picture consistent with the experimental observations reported in Ref. 11, assuming that, as supported by the measurements of the nanotube diameter, for the most part the $\mathrm{CN}$ contained in the zeolite matrix have preferently a $(3,3)$ geometry. We have shown that the $(5,0)$ nanotubes have an extended phase with strong CDW correlations, while the relevant instability for the $(3,3)$ geometry is given by the breakdown of the Luttinger liquid behavior as the renormalized velocity $v_{J_{+}}$goes to zero. This effect accounts for the appearance of a gap in the electronic spectrum as well as for an enhanced susceptibility in the electronic current, consistent with a large diamagnetic signal. These are signatures of a genuine phase transition, which takes place without the breakdown of a continuous symmetry, and should be tested in further experimental studies of the smalldiameter nanotubes.
${ }^{1}$ L. Balents and M. P. A. Fisher, Phys. Rev. B 55, R11973 (1997).

${ }^{2}$ R. Egger and A. O. Gogolin, Phys. Rev. Lett. 79, 5082 (1997); Eur. Phys. J. B 3, 281 (1998).

${ }^{3}$ C. Kane, L. Balents, and M. P. A. Fisher, Phys. Rev. Lett. 79, 5086 (1997).

${ }^{4}$ A. A. Odintsov and H. Yoshioka, Phys. Rev. Lett. 82, 374 (1999); Phys. Rev. B 59, R10457 (1999).

${ }^{5}$ M. Bockrath et al., Nature (London) 397, 598 (1999).

${ }^{6}$ Z. Yao et al., Nature (London) 402, 273 (1999).

${ }^{7}$ A. Yu. Kasumov et al., Science 284, 1508 (1999).

${ }^{8}$ A. F. Morpurgo et al., Science 286, 263 (1999).

${ }^{9}$ J. González, Phys. Rev. Lett. 87, 136401 (2001).

${ }^{10}$ M. Kociak, A. Y. Kasumov, S. Gueron, B. Reulet, I. I. Khodos, Y. B. Gorbatov, V. T. Volkov, L. Vaccarini, and H. Bouchiat, Phys. Rev. Lett. 86, 2416 (2001); A. Kasumov, M. Kociak, M. Ferrier, R. Deblock, S. Gueron, B. Reulet, I. Khodos, O. Stephan, and H. Bouchiat, Phys. Rev. B 68, 214521 (2003).

${ }^{11}$ Z. K. Tang et al., Science 292, 2462 (2001).

12 J. González, Phys. Rev. Lett. 88, 076403 (2002); Phys. Rev. B 67, 014528 (2003).

${ }^{13}$ J. V. Alvarez and J. González, Phys. Rev. Lett. 91, 076401 (2003).

${ }^{14}$ H. J. Liu and C. T. Chan, Phys. Rev. B 66, 115416 (2002).
${ }^{15}$ Yu. A. Krotov, D.-H. Lee, and S. G. Louie, Phys. Rev. Lett. 78, 4245 (1997).

${ }^{16}$ J. Voit, Rep. Prog. Phys. 58, 977 (1995).

${ }^{17}$ The out-of-plane optical modes play a dominant role in $\mathrm{CN}$ of short radius, followed by in-plane optical modes and breathing modes, according to R. Barnett, E. Demler, and E. Kaxiras, Phys. Rev. B 71, 035429 (2005); See also A. De Martino and R. Egger, Phys. Rev. B 67, 235418 (2003).

${ }^{18}$ A. Sédéki, L. G. Caron, and C. Bourbonnais, Phys. Rev. B 65, 140515(R) (2002).

${ }^{19}$ D. W. Wang, A. J. Millis, and S. Das Sarma, Phys. Rev. B 64, 193307 (2001).

${ }^{20}$ P. Hawrylak, G. Eliasson, and J. J. Quinn, Phys. Rev. B 37, 10187 (1988).

${ }^{21}$ A. A. Nersesyan and A. M. Tsvelik, Phys. Rev. B 68, 235419 (2003).

${ }^{22}$ K. Kamide T. Kimura, M. Nishida, and S.Kurihara, Phys. Rev. B 68, 024506 (2003).

${ }^{23}$ J. González, F. Guinea, and M. A. H. Vozmediano, Nucl. Phys. B 424, 595 (1994).

${ }^{24}$ R. Saito, G. Dresselhaus, and M. S. Dresselhaus, Physical Properties of Carbon Nanotubes (Imperial College Press, London 1998), Chap. 6. 